\title{
EXPERIMENTAL INVESTIGATIONS OF LONGITUDINAL AND TWISTED TAPE INSERTS FOR AUGMENTATION OF HEAT TRANSFER
}

\author{
CH. V. K. N. S. N. MOORTHY ${ }^{1}$, V. SRINIVAS ${ }^{2}$, K. BHARADWAJAN ${ }^{3} \&$ S. V. D. PRASAD ${ }^{4}$ \\ ${ }^{1,3,4}$ Department of Mechanical Engineering, Institute of Aeronautical Engineering \\ (Autonomous), Dundigal, Hyderabad, Telangana State, India \\ ${ }^{2}$ Department of Mechanical Engineering, GITAM University, Vishakhapatnam, Andhra Pradesh, India
}

\begin{abstract}
In the past decade, heat transfer enhancement technology was developed and widely applied to heat exchanger applications like refrigeration, automotives, process industry, solar water heater etc. There has been a great attempt to reduce the size and cost of the heat exchanger, and energy consumption. The most significant variable in reducing the size and the cost of the heat exchanger is the heat transfer coefficient and pressure drop. At present, the technology of the Twisted-Tape Inserts ${ }^{[1]}$ to heat exchangers is widely used in various industries. Insertion of twisted tapes in a tube provides a simple passive technique for enhancing the convective heat transfer by introducing swirl into the bulk flow, and by disrupting the boundary layer at the tube surface, due to repeated changes in the surface geometry.

The present work is a practical attempt made to study the influences of the twisted tape inserts on the heat transfer coefficient $(h)$ and friction factor $(f)$ in a double pipe $U$ bend heat exchanger. The experimental work is done by considering various twisted tapes with different Twist Ratios from 5 to 20. The effect of inserts is studied with the variation of Reynolds Number (Re) from 3000 to 30000. The main objectives of the work include estimation of heat transfer and friction factor in double U-bend heat exchanger, comparison of the heat transfer and friction factor for counter flow and parallel flow in the double pipe U-bend heat exchanger, understanding the effect of twisted tape insert inside the flow for heat transfer augmentation and friction factor, comparison of the heat transfer with and without twisted tape inserts.
\end{abstract}

KEYWORDS: Double U-Bend Heat Exchanger, Heat Transfer Augmentation \& Friction Factor

Received: Apr 04, 2017; Accepted: May 13, 2017; Published: May 27, 2017; Paper Id.: IJMPERDJUN201721

\section{INTRODUCTION}

If an engineer is to install an 'advanced' exchange, it must have obvious advantages over the standard shell and tube unit. The three areas of interest here are reliability, safety, and cost. Heat transfer enhancement devices are commonly employed to improve the performance of already existing heat exchangers, or to reduce the size and cost of a proposed heat exchanger. An alternative goal is to use such techniques to increase the system's thermodynamic efficiency, which facilitates reduction in cost. Enhancement Techniques ${ }^{[1]}$ can basically be classified as passive and active techniques. The latter requires extra external power sources like, mechanical aids, surface fluid vibration, injection and suction of the fluid, jet impingement, and use of electrostatic fields. The former use surface modification or an additional device incorporated into the channel. These methods do not require any direct application of external power. Typical examples of passive augmentation techniques are surface roughness, displaced promoters, and vortex generators. Surface roughness has been used to reduce the thickness of 
the boundary layer ${ }^{[2]}$ close to the surface. This enables better fluid mixing. Displaced promoters include inserts that alter the flow mechanism near the surface by disturbing the core flow. The vortex can be created through coiled wires, stationary propellers, or twisted tapes. The enhancement of a single phase flow is important because back flow usually represents the dominant thermal resistance in a two fluid heat exchanger, especially if it is a gas, as compared to a liquid.

Due to the many variables involved ${ }^{[8]}$, selecting optimal heat exchangers is challenging. Hand calculations are possible, but much iteration is typically needed. As such, heat exchangers are most often selected via computer programs, either by system designers, who are typically engineered, or by equipment vendors. In order to select an appropriate heat exchanger, the system designers (or equipment vendors) would firstly consider the design limitations for each heat exchanger type. Although cost is often the first criterion evaluated, there are several other important selection criteria which include High/ Low pressure limits, Thermal Performance, Temperature ranges, Product Mix (liquid/liquid, particulates or high-solids, liquid), Pressure Drops across the exchange, Fluid flow capacity, Clean ability, maintenance and repair, Materials required for construction, Ability and ease of future expansion. Choosing the right heat exchanger (HX) requires some knowledge of the different heat exchanger types, as well as the environment in which the unit must operate. Typically in the manufacturing industry, several differing types of heat exchangers are used for just the one process or system to derive the final product. For example, a kettle HX for pre-heating, a double pipe HX for the 'carrier' fluid and a plate and frame HX for final cooling. With sufficient knowledge of heat exchange types and operating requirements, an appropriate selection can be made to optimize the process.

During the present experiment, the annulus surface was assumed to be at a constant temperature throughout, which is approximated to be the average of the inlet and outlet temperature of the hot fluid (annulus fluid). The main objectives of the work include estimation of heat transfer and friction factor in double U-bend heat exchanger, comparison of the heat transfer and friction factor for counter flow and parallel flow in the double pipe U-bend heat exchanger, understanding the effect of twisted tape insert inside the flow for heat transfer augmentation and friction factor, comparison of the heat transfer with and without twisted tape inserts.

\section{EXPERIMENTAL SETUP AND OPERATIONAL PROCEDURE}

\section{Experimental Setup}

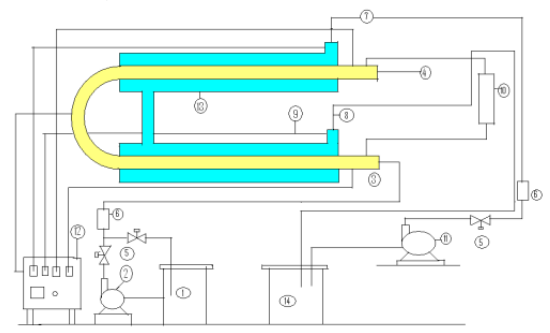

Figure 1: Plain U-Bend Double Pipe Heat Exchanger

The experimental test section consists of a shell and tube type heat exchanger with inlet and outlet passages at the end of the core and shell with six thermocouple provisions at the inlet and outlet of core and shell, respectively. An arrangement is provided at the end of the core to insert longitudinal and twisted tapes. The core pipe is about $19 \mathrm{~mm}$ in diameter, $1 \mathrm{~mm}$ thick and made of stainless steel. The shell is of around 1.5 inch diameter, and $1 \mathrm{~mm}$ thick, made of galvanized iron. The height of the test section is $0.35 \mathrm{~m}$, the length of the core and shell are so managed that the effective 
heat transfer path for the tube and shell should be $1.6 \mathrm{~m}$. So, the length is maintained that the flow through the tube is fully developed flow, so that during the flow entire cross section of the pipes should be filled with fluid.

\section{Annulus Fluid Circuit}

The elements of the annulus fluid circuit include Tank for hot fluid, Pump, Bypass valve arrangement, Test section.

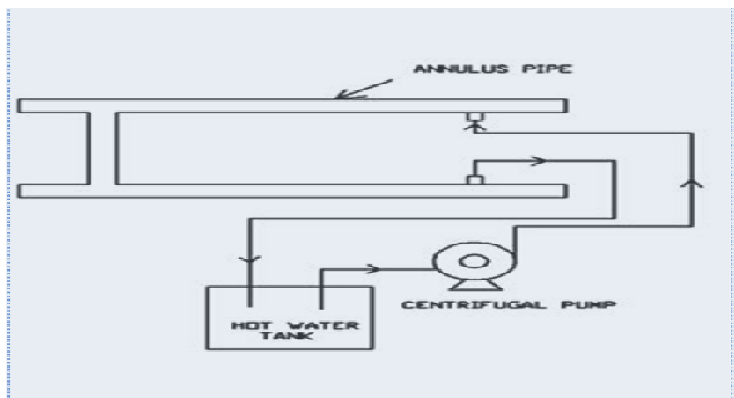

Figure 2: Line Diagram of Annulus Fluid Circuit

Water in the tank is initially heated to around 85 to $90^{\circ} \mathrm{C}$ by means of auxiliary heaters. This is then supplied to the test section through fiber reinforced PVC pipes to the test section. A bypass valve arrangement is provided, so that only the required amount of fluid enters the test section and the rest is deposited back into the tank. The out coming fluid from the test section is again re-circulated into the tank, where a large pool of water at a higher temperature is available and the temperature drop during the flow through the shell is compensated to some extent. The heating is again continued by auxiliary heaters so that the fluid in the tank is always maintained at a constant temperature throughout the experiment. The fluid employed here in the experiment is pure water as the properties of raw water always differ from that of pure water. An asbestos rope is wound throughout the heat transfer path of the shell to avoid loss of heat to the surrounding of the hot fluid.

\section{Core Fluid Circuit}

The elements of the core fluid circuit include Tank for cold fluid (core fluid), Pump, Bypass valve arrangement, and Test section

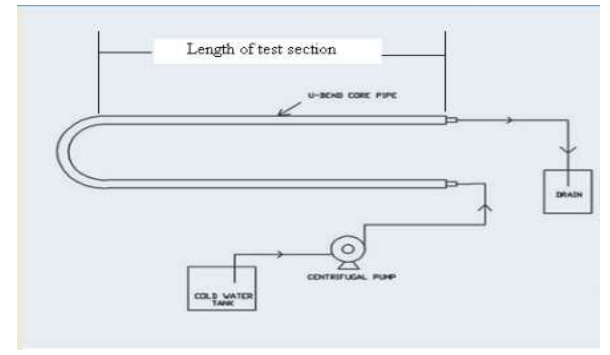

Figure 3: Line Diagram of Core Fluid Circuit

Cold fluid from the reserve tank enters the test section through bypass valve regulation and the out coming fluid from the test section will be drained out. Enough care is taken that the temperature of the hot fluid is always maintained constant. Six thermocouples (p, type) are located at the inlet and outlet of the core and shell. The leads of the thermocouples are connected to a temperature indicator and the values of the temperatures are tabulated. A measuring jar 
of 1 liter capacity is made use of for measuring the mass flow rate through the core and shell, and the time taken to collecting of 1 liter of the fluid is noted down by using a stop watch.

\section{Experimental Fabrication}

A stainless steel tube is taken and bent into a $U$ shape to the required size and shape. The annulus pipe is arranged at the upper and lower section of the stainless steel tube. Brazing is made at both ends of upper and lower ends of the pipe. Six thermocouples are placed, four at the ends of both the pipes and rest two in the middle of the Annulus pipe. The annulus pipe is wound by the insulation (asbestos rope). The whole test section is placed in the constructed frame as per the schematic diagram. The test section has an inlet and outlet flows which are connected by the PVC pipes. Reservoir tanks are arranged (hot fluid tank, cold fluid tank) at the base of the constructed frame, hence a bypass arrangement and flow meter are placed between the inlets of the test section and the reservoir tank, and outlet flows are arranged to re-circulate the fluids from the reservoir tank to the test section and vice versa. The setup is present such that the fluid from the reservoir tank flows into the test section with the help of a centrifugal pump and back into the reservoir tank.

\section{Experimental Procedure}

First, the plain tube double pipe heat exchanger is tested. At the beginning of test series, the hot water is circulated through annulus tube and cold water through the core tube in the counter flow configuration. The flow rate of hot water is fixed constant to $5.7 \mathrm{lit} / \mathrm{min}$. The cold water coming into the heat exchanger is at room temperature and flow rate of cold water is varied. Usually, $1 / 2$ hour was required for the attainment of steady state. Once the steady state is reached flow rate of hot water is kept constant and with varying cold water flow rates, at various Reynolds numbers, the temperature reading of the inlet and outlet section of cold and hot fluids and intermediate temperatures along with pressure drop reading were taken.

After completing the experiment with plain heat exchangers, the longitudinal strip was inserted into both the straight legs of the u-tube and above procedure was repeated for different flow rates of cold fluid.

After completing the experiment with longitudinal strip inserts, the strip is removed from the tube and half length twisted tape was inserted into both the straight legs of the u-tube, and the above procedure was repeated for different flow rates of cold fluid.

After completing the experiment, the range of values of various parameters in the present investigation was considered for finding the heat transfer rate. First, the heat input is calculated by different flow rates and temperature difference. The average wall temperature and the bulk mean temperature were combined with heat flux to get Nusselt Number ${ }^{[10]}(\mathrm{Nu})$, all the fluid properties are evaluated at the mean film temperature. Pressure drop data is calculated under isothermal condition.

\section{Assumptions}

Fin effect is neglected, Pressure gradient is assumed as constant, the constant volume flow rate of hot water, a closed loop system of hot water flow.

\section{Precautions}

The experiment is done by the circulation of water (properties dependent), mass flow rate is maintained constant for accurate results, An error analysis before the start of the experiment will prevent the errors generally found due to 
wrong connections of thermocouples, To avoid heat loss the outer tube is insulated with asbestos ropes.

\section{RESULTS AND DISCUSSIONS}

Graphs for Plain Tube Heat Exchanger

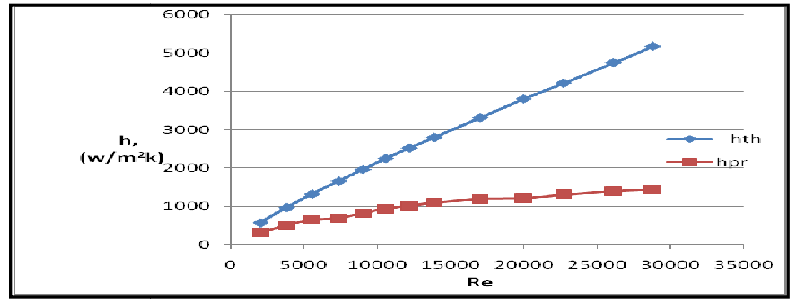

Figure 4: Effect of Re on h for Counter Flow Heat Exchanger

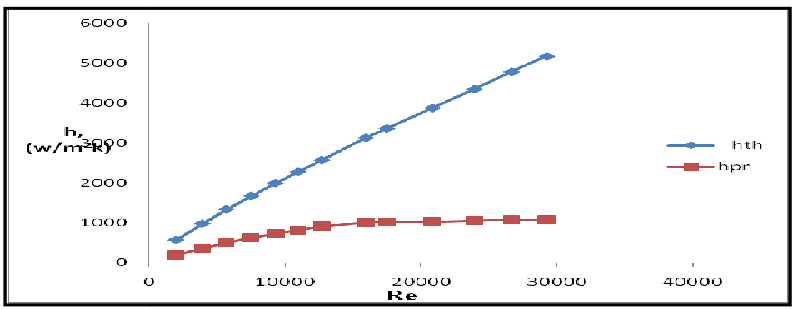

Figure 5: Effect of Re on h for Parallel Flow Heat Exchanger

Figure $4 \& 5$ show that the heat transfer coefficient increases with increasing the Reynolds number. Also, observed that the theoretical heat transfer coefficient is increasing linearly, when compared to that of practical heat transfer coefficient, due to varying flow rates and the conductivities of the annulus and shell material.

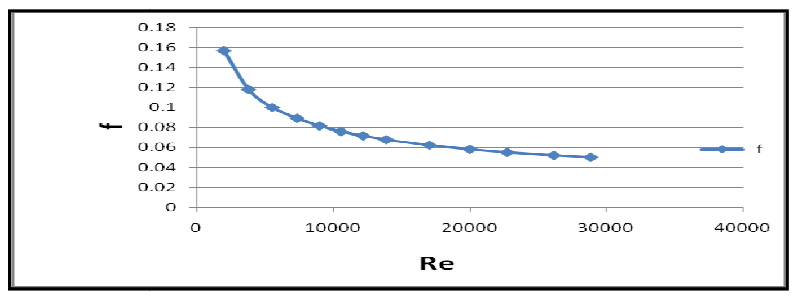

Figure 6: Effect of Re on for Counter Flow Heat Exchanger

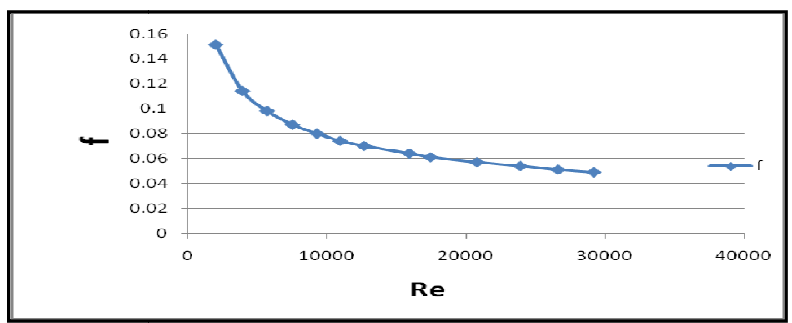

Figure 7: Effect of Re on f for Parallel Flow Heat Exchanger

Figure $6 \& 7$ show friction factor decreases with increase in Reynolds number. The Friction factor depends not only on Reynolds number, but also on the roughness of the pipe surface. The Friction factor is a minimum for smooth surface and increases with increasing roughness. The Reynolds number is a function of velocity. Friction factor decreases with the increasing of velocity. 


\section{Comparison of Heat Transfer Coefficient between Counter}

Flow and Parallel Flow Heat Exchangers

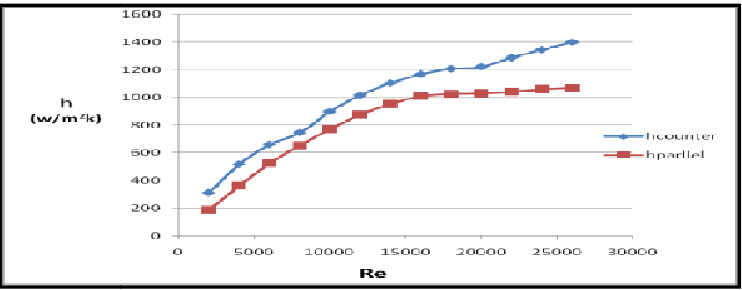

Figure 8: Effect of Re on h between Counter Flow and Parallel Flow Heat Exchanger

Figure 8 shows the heat transfer coefficient between the counter and parallel flow. The rate of increase of the heat transfer in case of counter flow is higher than that of parallel flow for a given Reynolds number. This is because the LMTD

${ }^{[10]}$ for a counter flow heat exchange is more than that of a parallel arrangement flow in the same temperature field for a given heat transfer rate.

\section{Graphs for Longitudinal Inserts}

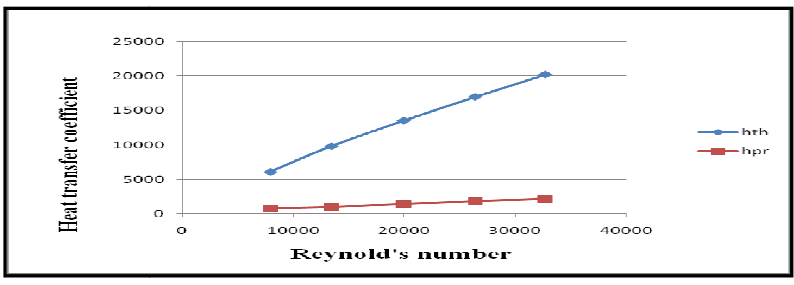

Figure 9: Effect of $\operatorname{Re}$ on $h$ for $A R=1$

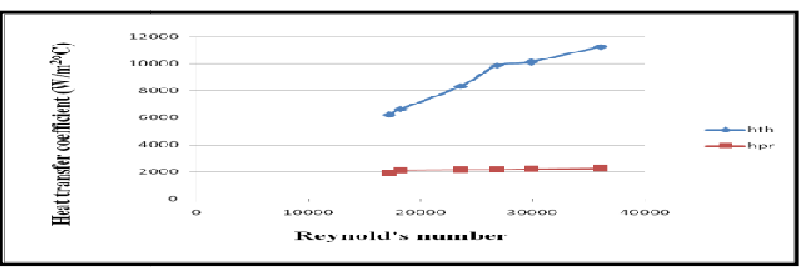

Figure 10: Effect of $\operatorname{Re}$ on $h$ for $A R=2$

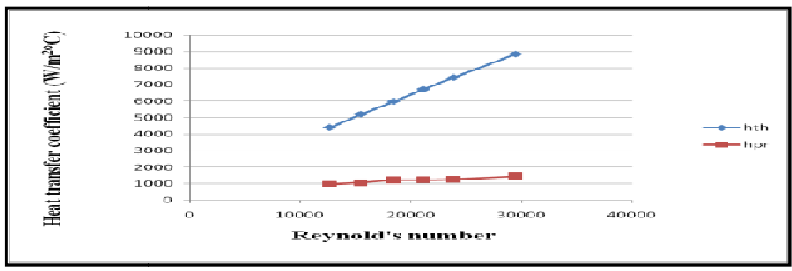

Figure 11: Effect of $\operatorname{Re}$ on $h$ for $A R=4$ 


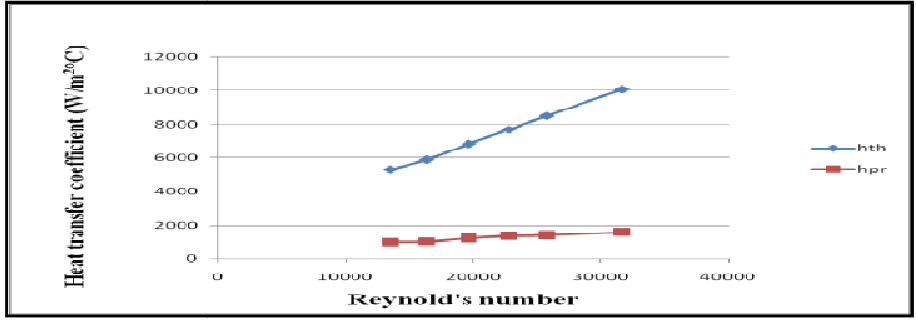

Figure 12: Effect of $\operatorname{Re}$ on $h$ for $A R=12.33$

From Figure 9,10,11 \& 12 it is observed that there is an increase in heat transfer coefficient, with increasing Reynolds number. This is due to the increase in the mass flow rate of the cold water with the increase in Reynolds's number. It is observed that the theoretical heat transfer coefficient increases linearly, when compared to the practical heat transfer coefficient. The variations are due to varying flow rates and the conductivities of the annulus and shell material. Uniform and constant temperature of the wall of the annulus is practically impossible.

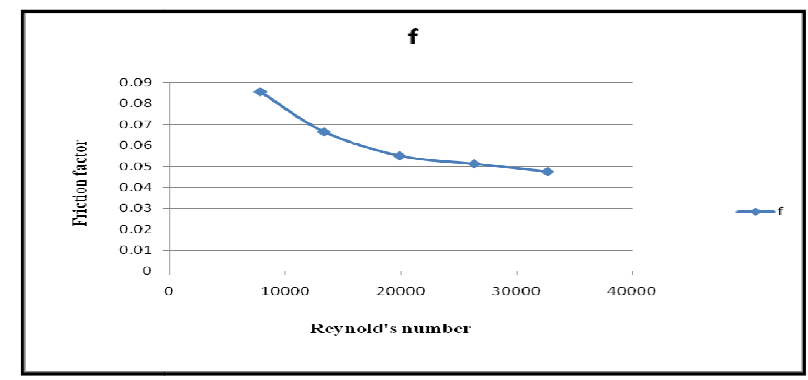

Figure 13: Effect of $\operatorname{Re}$ on $f$ for $A R=1$

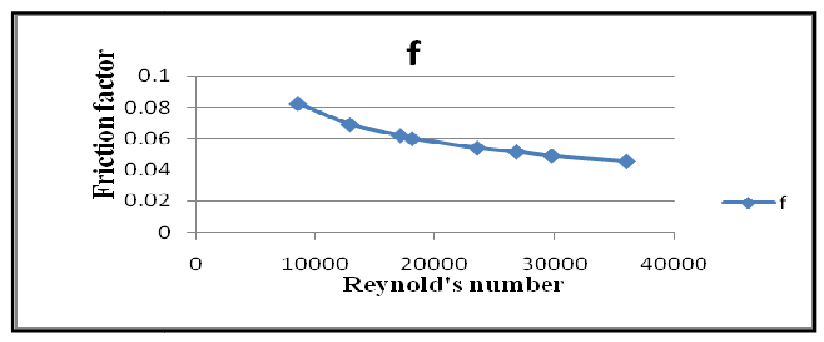

Figure 14: Effect of $\operatorname{Re}$ on $\mathrm{A} A \mathrm{R}=2$

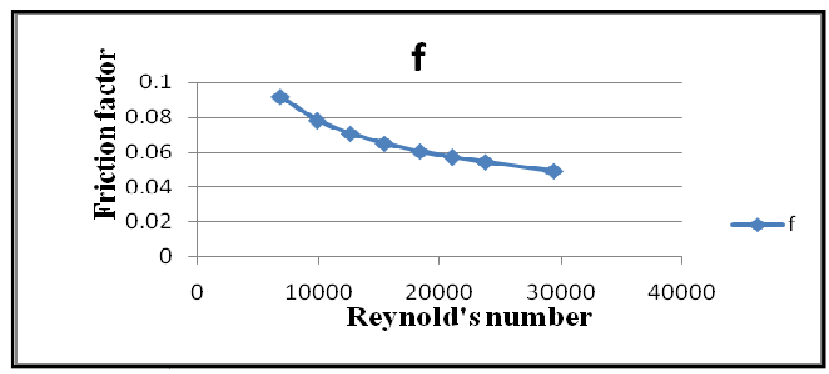

Figure 15: Effect of $\operatorname{Re}$ on $f$ for $A R=4$ 


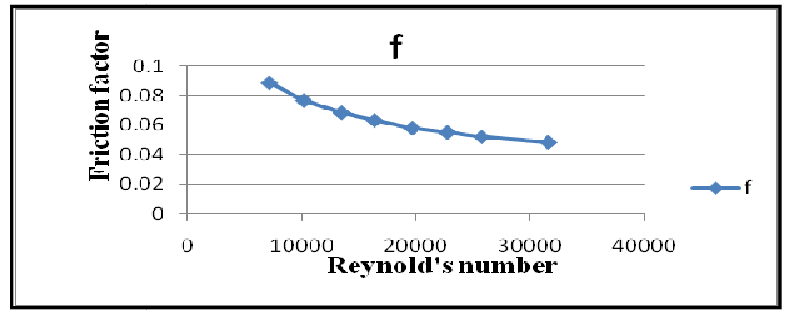

Figure 16: Effect of $\operatorname{Re}$ on $f$ for $A R=12.33$

From figure 13, $1415 \& 16$, it can be seen that friction factor decreases with increase in Reynolds number. The friction factor depends not only on Reynold's number, but also on the roughness of the pipe surface. The friction factor is minimum for a smooth surface and increases with increasing roughness. Reynold's number is a function of velocity. Friction factor decreases with an increase in velocity.

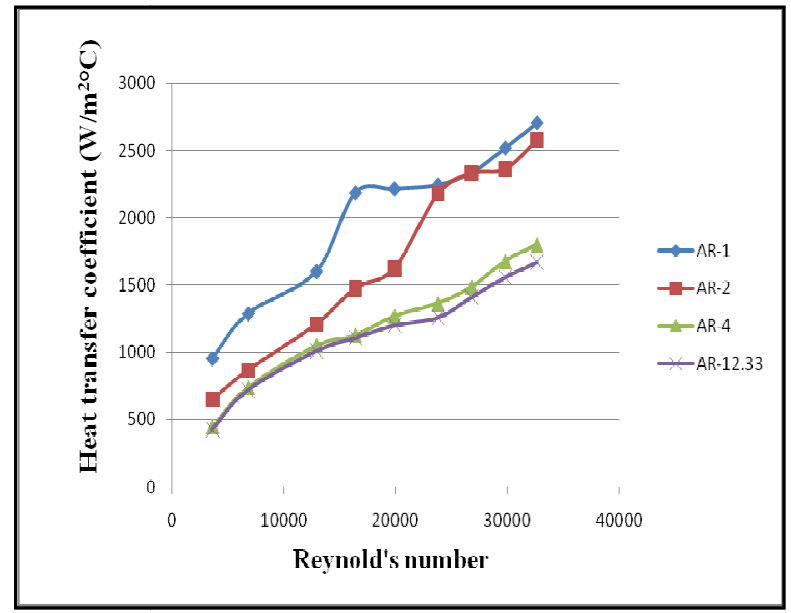

Figure 17: Comparision of $h$ for different ARs 1,2,3 and 12.33

A comparison study is made on the heat transfer coefficient for different aspect ratios, and it is observed that the heat transfer coefficient is higher for lower values of aspect ratios as shown in Figure 17. Increase in aspect ratio leads to a decrease in hydraulic diameter, which in turn increases the velocity, therefore resulting in an increase in the Reynolds's number. This leads to an increase in heat transfer with a decrease in aspect ratio. 


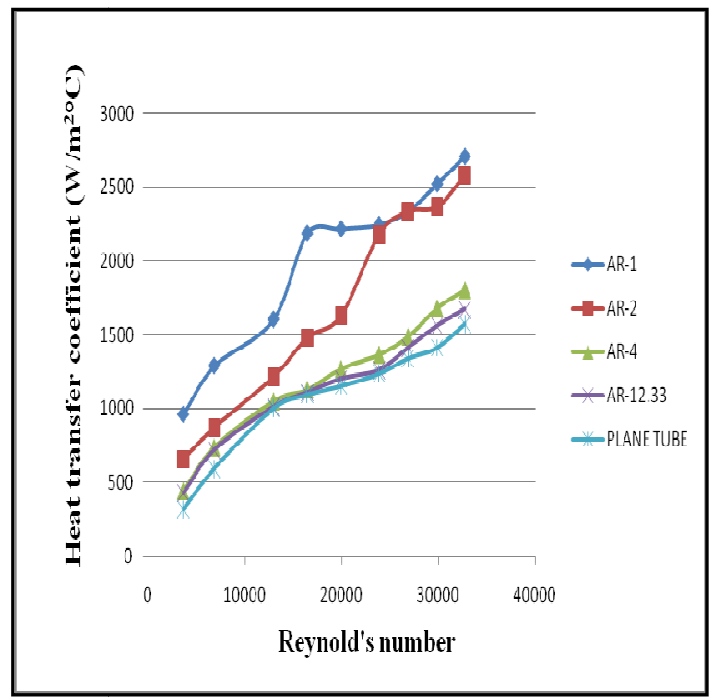

Figure 18: Comparision of $h$ with and with Out Strips

A comparison study is made in the heat exchanger with and without stripes. Higher values of heat transfer coefficient are obtained in the heat exchanger with strips as compared to the case, where strips are not used as shown in Figure 18.

\section{Graphs for Twisted Tape Inserts}

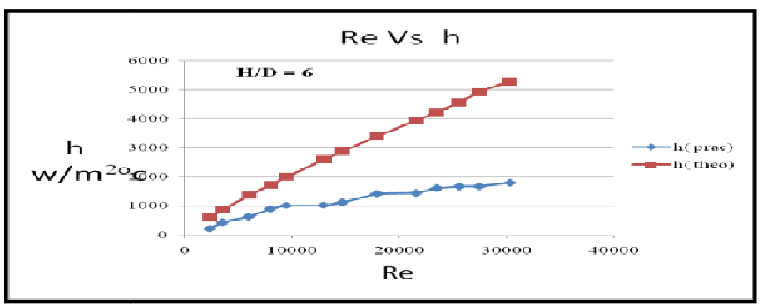

Figure 19: Effect of $\operatorname{Re}$ on $h$ for $H / D=6$

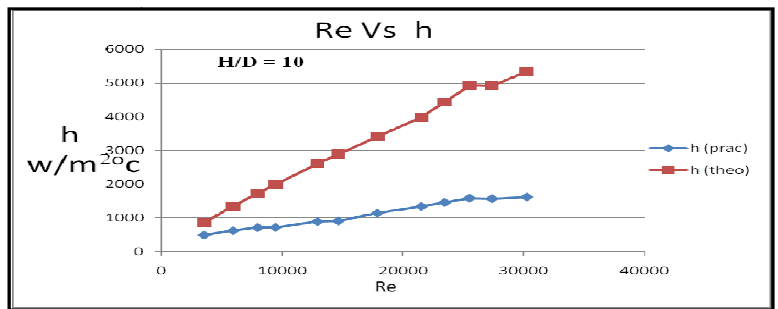

Figure 20: Effect of $\operatorname{Re}$ on $h$ for $H / D=10$

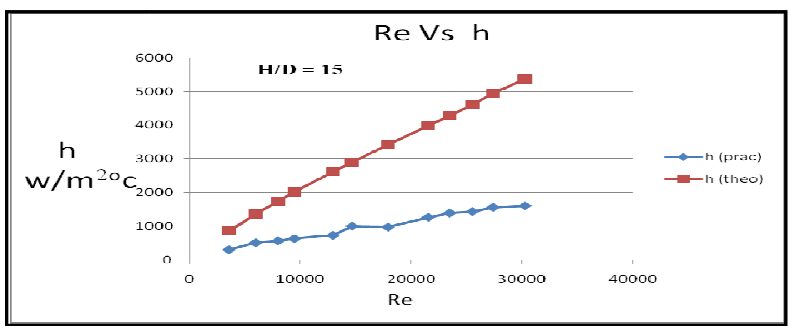

Figure 21: Effect of $\operatorname{Re}$ on $h$ for $H / D=15$ 


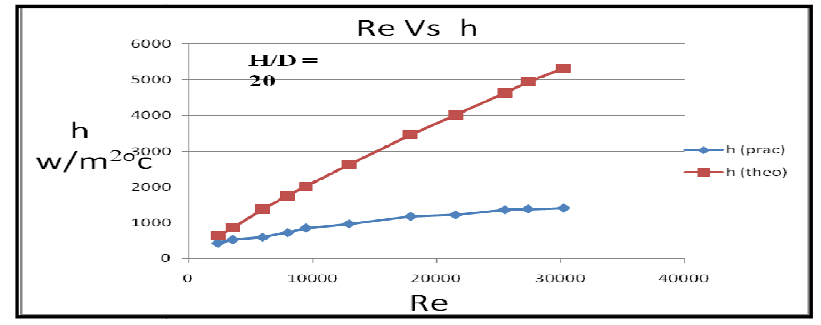

Figure 22: Effect of $\operatorname{Re}$ on $\mathrm{h}$ for $\mathrm{H} / \mathrm{D}=\mathbf{2 0}$

From figure 19, 20, $21 \& 22$, it is observed that Heat transfer coefficient increases with increase of Reynolds number. This is due to the increasing in the mass flow rate of the cold water.

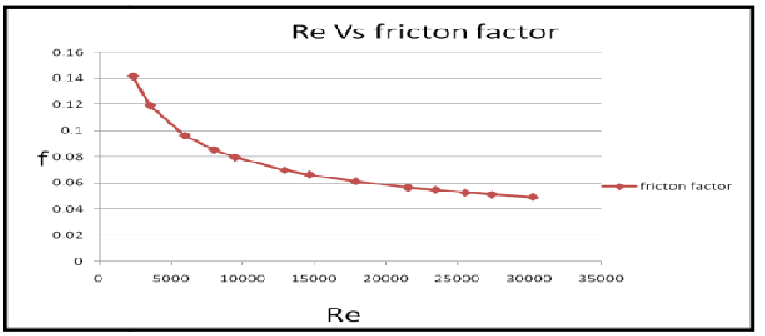

Figure 23: Effect of Re on f for all Twist Ratios

Here in Figure 23 it has been observed that the the friction factor decreases while the Reynolds number increases. This is due to the friction factor, a function of velocity; if the velocity increases the friction factor decreases, friction is coming in to picture when the velocity of the fluid is less.

\section{Comparison between different Twist Ratios}

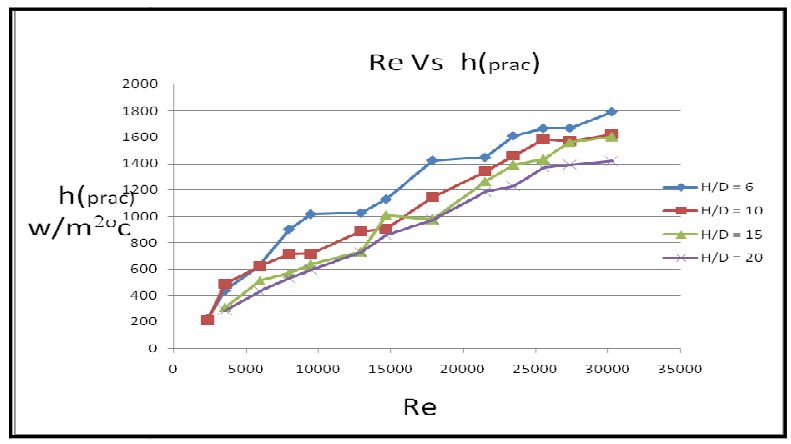

Figure 24: Comparison between different H/D Ratios

In Figure 24 the graph is drawn between the effect of heat transfer coefficient on Reynolds number for different twist ratios. It found that the less H/D ratio (i.e more number of turns) have more heat transfer coefficient than others twist ratios. This is due to the Recident time of the fluid particle is increased. 
Comparison Between Twist Insert with H/D = 6 and Longitudinal Strips of Different Aspect Ratio

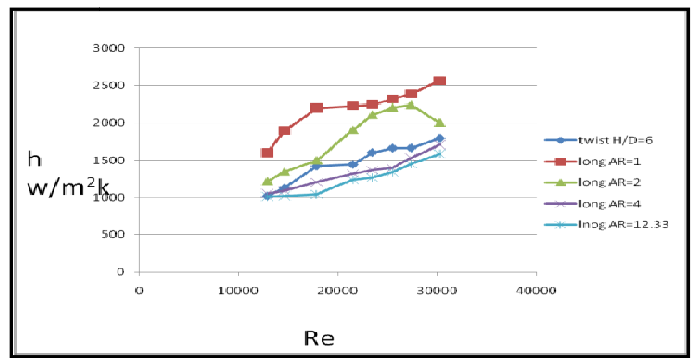

Figure 25: Effect of $\operatorname{Re}$ on $h$ for $H / D=6$ and Longitudinal Strips

In figure 25, itt is observed that the aspect ratios having 1 and 2 have more heat transfer coefficient than twisted tape 6. From this, we can conclude that the strips having AR 1 and 2 create more turbulence than twisted tape.

\section{CONCLUSIONS}

During the experiment, the annulus surface was assumed to be at a constant temperature throughout, which is approximated to be the average of the inlet and outlet temperature of the hot fluid (annulus fluid). The rate of heat transfer and friction factor in the U-bend heat exchanger is $9005 \mathrm{~J} / \mathrm{s}$ and 0.079 . The heat transfer coefficient increases with increase in Reynolds number, and friction factor decreases with increase in Reynolds number. For a given Reynolds number, the rate of increase of the heat transfer coefficient in case of counter flow is higher than that of parallel flow. The rate of heat transfer for the heat exchanger with longitudinal strips is $12 \%$ more than that of without strips. The friction factor with twisted tape inserts inside a flow in U-Bend heat exchanger is 0.050. The rate of heat transfer for the heat exchanger with twisted tapes is $15 \%$ more than that of without tapes. Our experiment is with twisted tapes of different twist ratios, from our results it is found that the tape having H/D ratio 6 having a high heat transfer coefficient than the tapes of H/D ratios 10, 15,20 . This is due the increase of the resident time of the fluid particle and turbulence.

\section{REFERENCES}

1. S.S.Joshi, V.M.Kriplani, "Review of Heat Transfer Augmentation with Tape Inserts", International Journal of Engineering Science and Technology, Voloume 3, Issue 3, March 2011, pp 1949-1952

2. J. P. Abraham, E. M. Sparrow, J.C.K. Tong, "Heat Transfer in All Pipe Flow Regimes - Laminar, Transitional/Intermittent, and Turbulent", International Journal of Heat and Mass Transfer, Vol. 52, pp. 4040-4046, 2009.

3. Lap MouTtam and Afshin J. Ghazar, "Transitional heat transfer in Plain Horizontal tubes", Heat transfer Engineering, 27(5):23-38, 2006.

4. Paisarn Naphon, "Heat transfer and pressure drop in the horizontal double pipes with and without twisted tape inserts", International Communications in Heat and Mass Transfer, Vol. 33, 2006, pp. 166-175

5. Eiamsa-ard, Nivesrangsan and Chokphoemphun. Promvonge, "Influence of combined non-uniform wire coil and twisted tape inserts on thermal performance characteristics”, International Communications in Heat and Mass Transfer, Vol. 37, 2010, pp. $850-856$

6. Jafari Nasr M.R., Habibi Khalaj A. and Mozaffari S.H., " Modeling of heat transfer enhancement by wire coil inserts using artificial neural network analysis", Applied Thermal Engineering, Vol. 30, 2010, pp.143-151

7. S.K. Agarwal, M. Rajarao, "Heat transfer augmentation for the flow of viscous liquid in circular tubes using twisted tape 
inserts”, Internet J. Heat Mass Transfer 39 (1996) 3547-3557.

8. Bergles.A.E., Techniques to Augment Heat Transfer, Hand book of Heat Transfer Applications., Ed. Roshenow W.M.et al., Chapter 3, Mc.Graw Hill, New York 1985.

9. K. Bensayah, A. Benchatti, M. Aouissi, A. Bounif, Scalar turbulence model investigation with variable turbulent Prandtl number applies in hot axisymmetric turbulent round jet, Heat Tech. 25 (2007) 49-56.

10. SACHDEVA, Fundamentals of Heat and Mass Transfer.

11. Sibel Gunes, Veysel Ozceyhan and Orhan Buyukalaca, "Heat transfer enhancement in a tube with equilateral triangle cross sectioned coiled wire insert”, Experimental Thermal Fluid Science, 2010, Vol. 34, Issue 6, pp. 684-691

12. Andreas Hantsch, Ulrich Gross and Andrew R. Martin, "Heat Transfer Augmentation: Radiative-Convective Heat Transfer in a Tube with Fiber Array Inserts", Journal of Heat Transfer, February 2010, Vol. 132, 023505-1 to 6.

13. Murugesan, K. Mayilsamy, S. Suresh, P.S.S. Srinivasan, "Heat transfer and pressure drop characteristics in a circular tube fitted with and without V-cut twisted tape insert", International Communications in Heat and Mass Transfer Volume 38, Issue 3, March 2011, Pages 329-334. 